\title{
Methods of ensemble prediction for seasonal forecasts with a coupled ocean-atmosphere model
}

$\begin{array}{lll}\text { J. S. Frederiksen } & \text { C. S. Frederiksen } & \\ & & \text { S. L. Osbrough } \\ & \end{array}$

(Received 6 December 2012; revised 15 June 2013)

\begin{abstract}
Computational methods for efficient seasonal ensemble prediction with a coupled ocean-atmosphere model, consisting of a global atmosphere and a Pacific basin ocean, are described. Nonlinearly modified Lyapunov vectors, termed bred modes, and finite time normal modes, termed cyclic modes, that grow fastest over a month are found to be suitable ensemble perturbations. The skill of seasonal ensemble prediction is examined in hindcast simulations for the period 1980 to 2000. In general, ensemble mean forecasts are significantly more skilful than the control forecasts. We find that cyclic mode perturbations are generally more effective than bred vectors in improving ensemble forecasts.
\end{abstract}

http://journal.austms.org.au/ojs/index.php/ANZIAMJ/article/view/6509 gives this article, (c) Austral. Mathematical Soc. 2013. Published July 9, 2013, as part of the Proceedings of the 16th Biennial Computational Techniques and Applications Conference. ISSN 1446-8735. (Print two pages per sheet of paper.) Copies of this article must not be made otherwise available on the internet; instead link directly to this URL for this article. 
Subject class: $76,60 \mathrm{G} 25$

Keywords: Fluid mechanics, Prediction theory

\section{Contents}

1 Introduction

C362

2 Coupled ocean-atmosphere model

C363

3 Theoretical approach

C364

3.1 Calculation of bred vectors and cyclic modes . . . . . . . C364

3.2 Growth rates and relative amplification factors . . . . . . C365

4 Analysis period

C366

$5 \quad$ Bred vector and cyclic mode coupled instabilities

C366

6 Seasonal ensemble forecasts

C367

6.1 Average forecast error growth . . . . . . . . . . C C369

6.2 Error growth variability during ENSO cycle . . . . . . . C371

6.3 Seasonal variability of error growth . . . . . . . . . C371

7 Conclusions

C373

References

C375

\section{Introduction}

Coupled ocean-atmosphere variability in the Pacific Ocean hemisphere associated with the quasi-periodic phenomenon known as El Niño-Southern Oscillation (ENSO) has a major impact on global climate variations over seasonal to interannual time scales. It also significantly affects the predictability 
in the tropical and subtropical regions. Recently, ensemble prediction methods were employed to improve the forecasts of weather [1,2] and seasonal climate variations [3]. Here we examine the properties of coupled ocean-atmosphere instabilities in the model described in the associated article by Frederiksen et al. (2013) [4] (hereafter FFB) and relate these properties to the boreal spring predictability barrier for seasonal prediction $[3,5,6]$. A second major aim is to examine whether ensemble forecasts with bred vectors or cyclic modes as ensemble perturbations yield improvements over control forecasts with the coupled ocean-atmosphere model of FFB. We examine the extent to which the improvements in prediction with ensemble forecasts depend on the number and type of ensemble perturbations. As well, we examine the variability in forecast skill during the ENSO cycle and during the average annual cycle.

In Section 2 we outline the coupled ocean-atmosphere model used in this study. Section 3 presents the theoretical approach employed for generating ensemble perturbations and Section 4 describes the method of obtaining the analyses for initialising the forecasts between 1980 and 2000. In Section 5 the growth properties of ensemble perturbations are described while in Section 6 the skill of ensemble and control forecasts is described. Concluding remarks are presented in Section 7.

\section{Coupled ocean-atmosphere model}

The model used in this study has a global atmosphere coupled through surface wind stresses and heat fluxes to a Pacific basin ocean extending from $90^{\circ} \mathrm{S}$ to $90^{\circ} \mathrm{N}$ (see Figure 1 of FFB [4]), and with no through flow in the southern ocean. The resolution of both components corresponds to a model grid of circa $2.3^{\circ}$ latitude and $3.75^{\circ}$ longitude. Full details of the model are given by FFB. 


\section{Theoretical approach}

\subsection{Calculation of bred vectors and cyclic modes}

The bred vector coupled instabilities employed in this article were obtained using the breeding method described by Toth and Kalnay [1] and Frederiksen et al. [2]. In the breeding method a small perturbation is added to the initial conditions for a given forecast. The unperturbed control and the perturbed forecasts are then performed. After a period of time the differences between the perturbed and control forecasts are calculated. The perturbation is then scaled to a suitable small amplitude, and control and perturbed forecasts are performed from the analysis and perturbed analysis at the new time. The process is continued and generally for several pairs of perturbations with equal and opposite structures at the time of each rescaling. Frederiksen et al. [2, Figure 1] provide a schematic of the method.

If perturbations are kept suitably small and the restart periods sufficiently short, then for a deterministic dynamical system the perturbation will eventually converge to the fastest growing Lyapunov vector [1, 2]. However, in our studies we are interested in the large scale slower growing nonlinearly coupled modes of the ocean-atmosphere system. For this reason the restart time is taken to be one month, which allows the large scale coupled modes to be generated [3]. We are primarily interested in modes for which there is a significant equatorial ocean perturbation that is coupled to a large scale atmospheric perturbation. For this reason we mask the ocean perturbation by $\cos ^{8} \phi$, where $\phi$ is latitude, at each monthly restart, but leave the atmospheric perturbations unchanged. This mask was chosen, after experimentation, to localize the ocean perturbations in the equatorial region. All the perturbation fields are rescaled proportionally so that the $50 \mathrm{~m}$ root mean square temperature is $0.1^{\circ} \mathrm{C}$ in the NINO3+ region at each restart time. Here the NINO3+ region ranges from $90^{\circ} \mathrm{W}$ to $150^{\circ} \mathrm{W}$ longitude and from $10^{\circ} \mathrm{S}$ to $10^{\circ} \mathrm{N}$ latitude. The scaling factor was chosen to optimize the skill of the ensemble forecasts. 
Cyclic modes are calculated in the same way as bred vectors but are iterated several times before being employed in the perturbed forecasts. That is, in each month the evolved perturbations are rescaled to the standard magnitude and returned to the beginning of the month for the next iteration of growth. Therefore, the cyclic modes are more characteristic of the flow regime of the particular month than are bred vectors, particularly if there are rapid changes in the flow regimes, such as in boreal spring. In this study, the cyclic modes are obtained by three iterations, which is sufficient to significantly improve the ensemble forecasts.

\subsection{Growth rates and relative amplification factors}

We denote by $\mathbf{x}(\tau, \mathbf{t})$ a vector of grid point values of any of the atmospheric or oceanic fields of a bred vector or cyclic mode initiated at time $t$ and evolved to time $\tau+t$. Further, we denote the root mean square of the vector $\mathbf{x}(\tau, \mathrm{t})$, which is our $\mathrm{L}_{2}$ norm, by $\|\mathbf{x}(\tau, \mathrm{t})\|$. Then, with the initial time $\tau_{0}=0$, the total amplification factor of a perturbation initiated at $t$ and evolved between $t+\tau_{0}=t$ and $t+\tau$ is

$$
A(\tau, t)=\|x(\tau, t)\| /\|x(0, t)\| .
$$

We then define the local total growth rate $\tilde{\omega}_{i}(t)$, averaged over a time interval $T_{30}$ that we take to be 30 days, by

$$
\tilde{\omega}_{i}(t)=\frac{1}{T_{30}} \log A\left(T_{30}, t\right) .
$$

Further, we define the grand average, or global growth rate, $\omega_{i}$ as the average value of $\tilde{\omega}_{i}(t)$ as $t$ ranges between $t_{0}=0$ and $T_{\max }$, the last initial condition for the forecasts. Thus,

$$
\omega_{i}=\frac{1}{T_{\max }} \int_{0}^{T_{\max }} \mathrm{dt} \tilde{\omega}_{i}(t) .
$$


We also define the local relative growth rate by

$$
\hat{\omega}_{i}(t)=\tilde{\omega}_{i}(t)-\omega_{i} .
$$

The local relative growth rate is related to $R(t)$, the relative amplification factor, through

$$
R(t)=\exp \left(\int_{0}^{t} d s \widehat{\omega}_{i}(s)\right),
$$

where $R(0)=1$. We need to examine the average local total growth rates and amplification factors over several years, $\left\langle\tilde{\omega}_{i}(t)\right\rangle$ and $\langle R(t)\rangle$, respectively.

\section{Analysis period}

The forecasts described in this article start from analyses with validity dates spanning 1 January 1980 to 1 December 2000. This period includes the major El Niño event of 1997 followed by the La Niña of 1998. The analyses are determined using a nudging scheme for data assimilation described by FFB [4] who noted the scheme produces flow fields that are close to observations but that are also consistent with the model; as a consequence, there are no shocks when the model is started from these analyses.

\section{Bred vector and cyclic mode coupled instabilities}

The global growth rates of the bred vectors and cyclic modes are very similar at 0.02 day $^{-1}$, corresponding to an e-folding time of 50 days. Figure 1 shows the average local total growth rate for the temperature fields of bred vectors (blue), and cyclic modes (thin black), and the relative amplification factor of cyclic modes (thick black), based on the global norm in the atmosphere and 
ocean. The results are based on an average over eight perturbations and the monthly integrations are started every three days between 1 January 1992 and 1 December 1999. The maximum relative amplification factor for both the cyclic mode (Figure 1) and for the bred vector (not shown) occurs in early boreal spring and has a magnitude of nearly twice the value in January. It then decreases in late boreal spring, attaining low values in boreal autumn and generally increasing values in winter. The minimum in the relative amplification factor occurs between October and December. The characteristics of the bred vector and cyclic mode shown in Figure 1, including the large amplitudes of the relative amplification factor in the first half of the year, the small values in boreal autumn and the increases in boreal winter, are very similar to those found by Frederiksen and Branstator for the finite time normal modes [5, Figure 4] and for the finite time principal oscillation patterns [6, Figure 5]. Again, we expect that the seasonal variability in the basic state circulation is responsible for the seasonality in the growth and amplitude of the coupled instabilities. In boreal spring the oceanic growth rates of the cyclic modes are larger than those of bred vectors. This is presumably because the cyclic modes are better able to capture the monthly instabilities when the basic state is changing rapidly in boreal spring. In turn, this may explain why cyclic modes result in greater improvements in ensemble forecasts, as discussed in the next section.

\section{Seasonal ensemble forecasts}

We performed one year forecasts with the coupled ocean-atmosphere model, mentioned briefly in Section 2 and in more detail by FFB [4]. These forecasts were started on the first day of each month between 1 January 1980 and 1 December 2000. The control forecasts start from analyses obtained from the nudging method of data assimilation, as described in Section 4 of FFB. For the ensemble forecasts, the control initial conditions are perturbed by the bred vectors or cyclic modes obtained as described in Section 3. Ensemble 

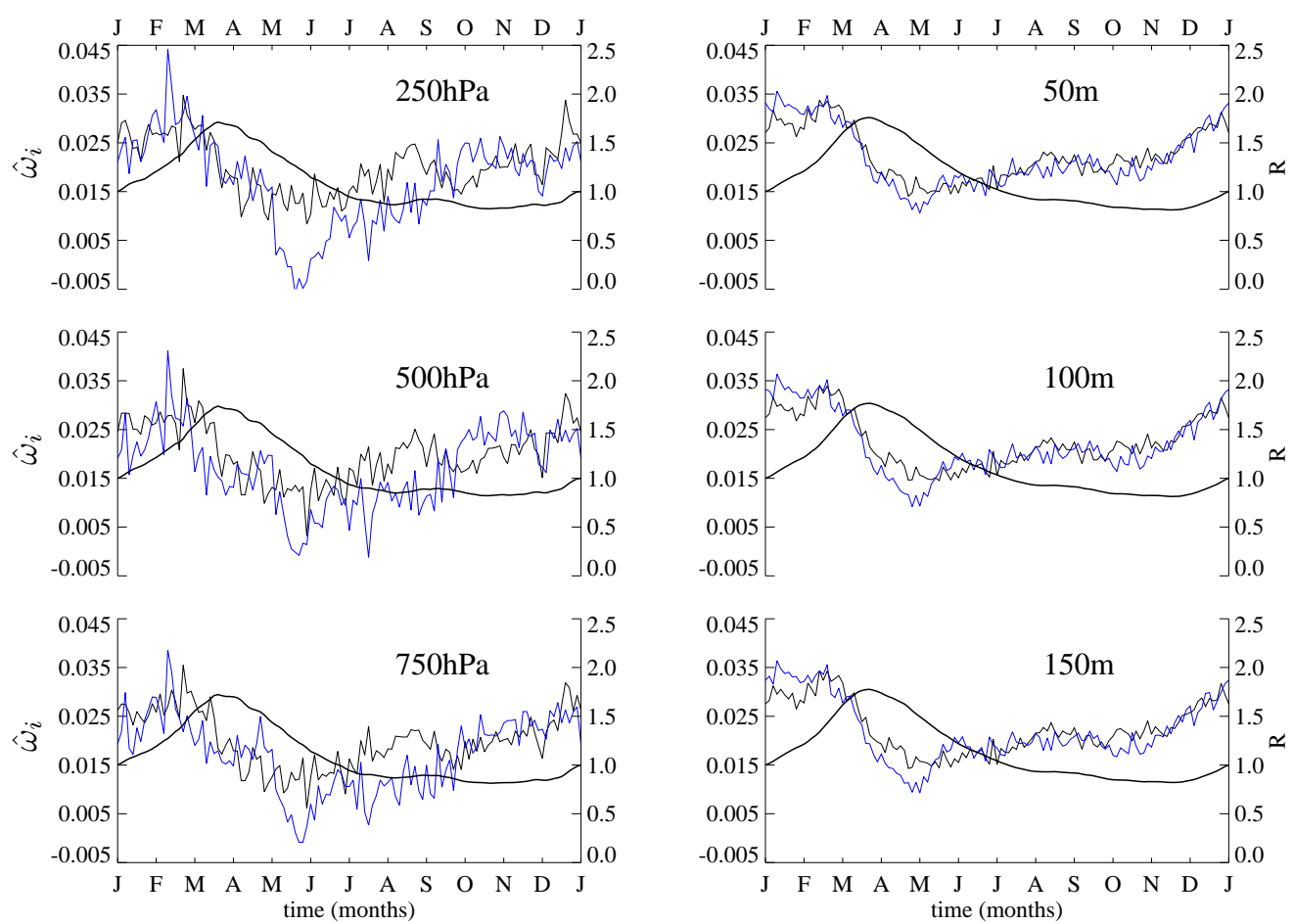

Figure 1: Bred vectors (blue), cyclic modes (thin black) and (Left) local total growth rate (day ${ }^{-1}$, thick black) at different atmospheric pressures; and (Right) relative amplification factor of cyclic mode (thick black) for temperature fields at different ocean depths. 
forecasts were performed with 2, 8, 32 and 64 paired perturbations; each member of a pair had the same structure but opposite sign. This ensures that the initial conditions of the ensemble mean are identical to those of the control. Our focus is on the skill of forecasts in predicting the $50 \mathrm{~m}$ ocean temperature for the NINO3+ region.

\subsection{Average forecast error growth}

We first consider how the root mean square (rms) forecast errors (forecast minus analysis) of the $50 \mathrm{~m}$ ocean temperatures of the NINO3+ region grow, on average, in 12 month ensemble and control forecasts. Figure 2(a) shows the mean amplification of rms errors over 12 months, where these errors were averaged over all forecasts which were started once a month between 1 January 1980 and 1 December 2000; the ensemble results are based on eight members. For forecasts longer than about two months the errors of the ensemble mean forecasts are smaller than for the control forecasts. Moreover, the relative improvement in the ensemble mean forecasts over the control grows with time until the errors saturate towards the end of the twelve month period. The sensitivity of the skill of ensemble forecasts to the number of members in the ensembles is deduced from Figure 2(b), which shows the difference between the control and ensemble mean forecast errors as a function of month and of the number of ensemble members. As expected, the larger the number of members the better the ensemble forecast, on average. Nevertheless, the improvement with increasing number of ensemble members saturates; there is little additional gain in using 64 members compared with using 32 members. Also, for forecast lead times between two and eight months ensemble prediction with eight cyclic modes is more skilful than even with 64 bred vectors. 


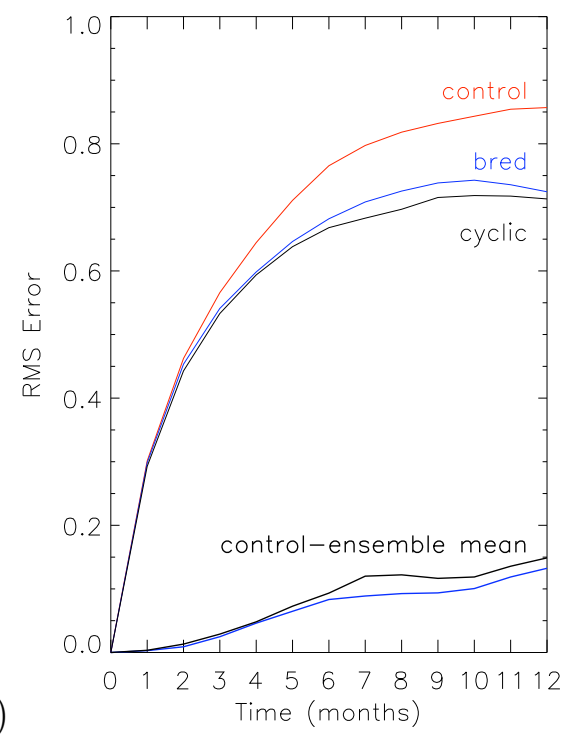

(a)

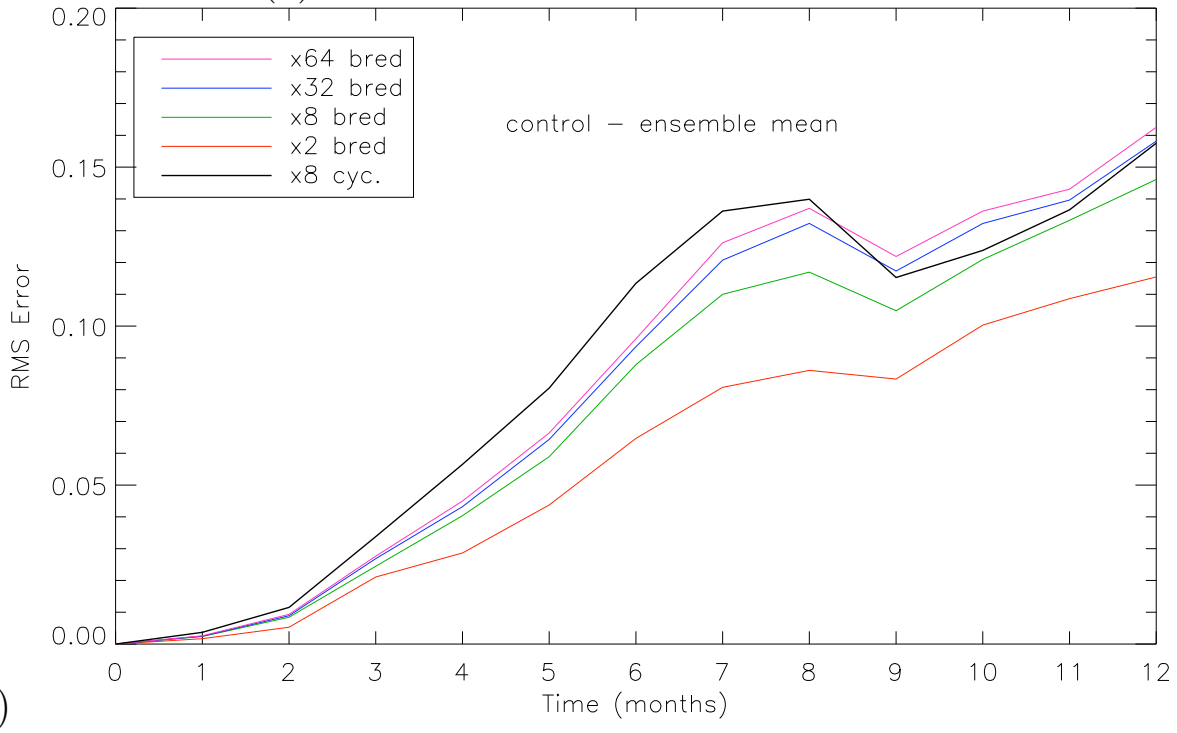

Figure 2: (a) Growth of rms errors of $50 \mathrm{~m}$ ocean temperatures $\left({ }^{\circ} \mathrm{C}\right)$ in the NINO3+ region as function of month (based on eight ensemble members). (b) Corresponding control minus ensemble mean errors for different numbers and types of ensemble perturbations. 


\subsection{Error growth variability during ENSO cycle}

We examine the variability of rms error growth during the period between 1 January 1990 and 1 December 2000 which includes the large El Niño event of 1997 and the La Niña event of 1998. Figure 3 shows the rms errors in $50 \mathrm{~m}$ ocean temperatures in the NINO3+ region for 12 month forecasts started each month. During this period there is considerable variability in rms errors of both control and ensemble mean forecasts, based on eight ensemble members, using cyclic modes. In particular, there is large variability in error amplitude during the El Niño event of 1997 and the subsequent La Niña event of 1998. Both the control and ensemble mean forecasts have difficulty in capturing the El Niño regime transition. Nevertheless, errors in the ensemble mean forecasts are generally reduced in comparison with the control forecasts and this reduction also applies to forecasts through the El Niño regime transition. Once the El Niño conditions are established then errors in both the control and ensemble mean forecasts are generally reduced. Forecasts through the La Niña transition also tend to be less skilful than in more quiescent periods with the ensemble mean forecasts having generally smaller errors than the control.

\subsection{Seasonal variability of error growth}

We examine the variability of forecast error growth during the annual cycle. We obtain the mean errors in a given month by averaging the forecasts errors in that month over all the years between 1990 and 2000. We display the results in Figure 4, which shows the annual cycle of error growth in the ensemble mean, based on eight cyclic mode ensemble members, and control forecasts, as well as the control minus ensemble mean difference, for rms errors in $50 \mathrm{~m}$ ocean temperatures in the NINO3+ region. Figure 4 shows the general tendency of errors to increase with time, as expected, and that there are peaks in amplitudes of errors for forecasts verified in boreal spring. The forecasts' error amplitudes show the characteristic boreal spring predictability 


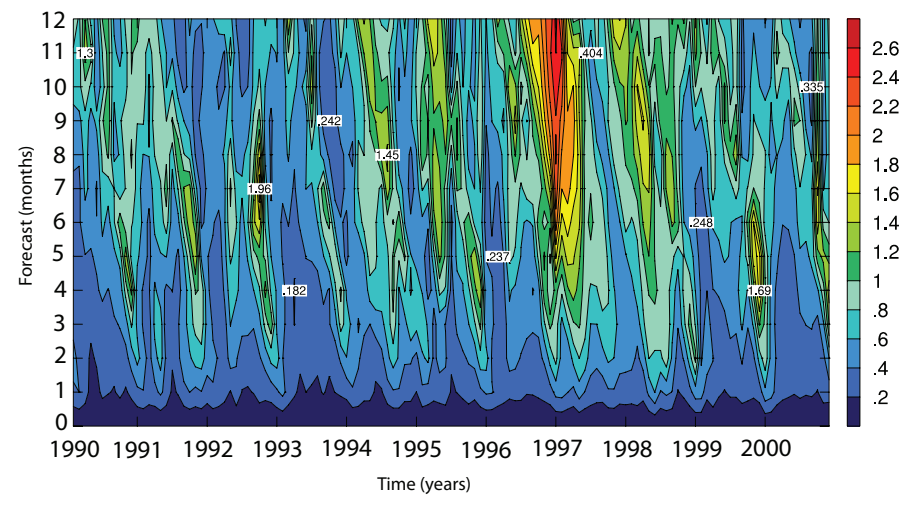

(a)

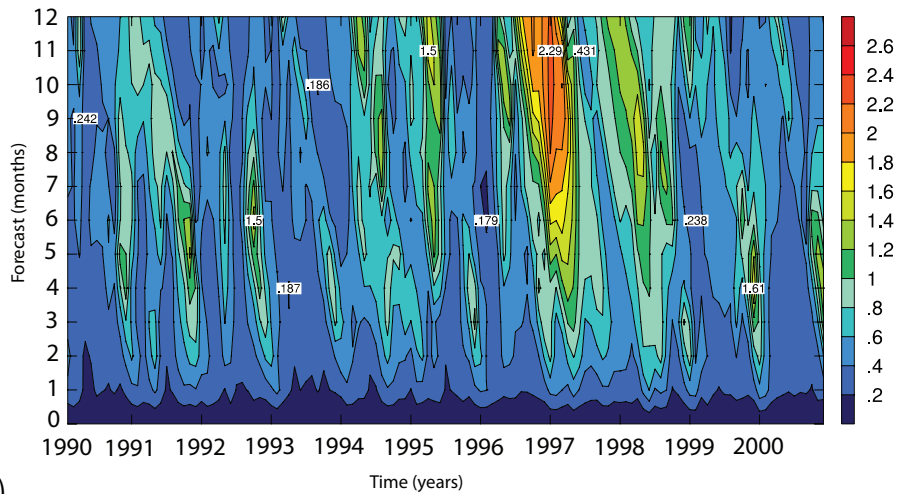

(b)

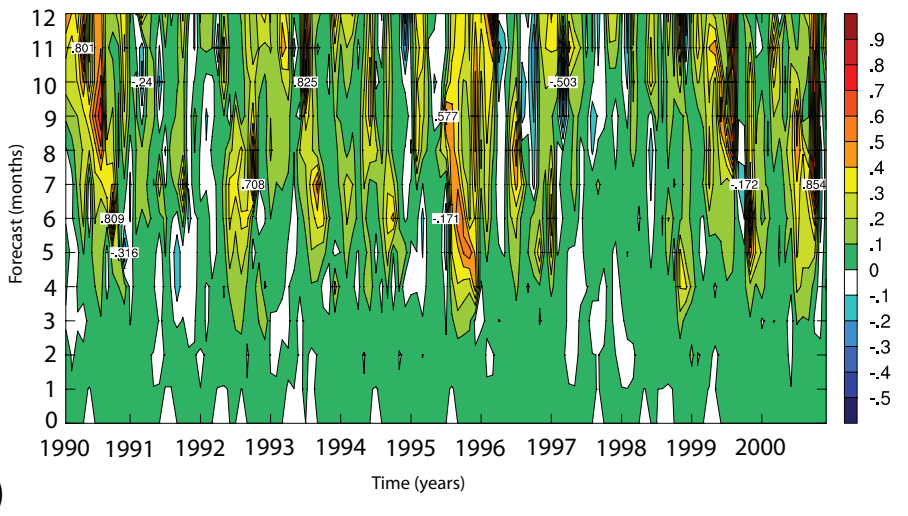

(c)

Figure 3: Growth of rms errors of $50 \mathrm{~m}$ ocean temperatures $\left({ }^{\circ} \mathrm{C}\right)$ in the NINO3+ region as function of forecast month, starting every month between January 1990 and December 2000 for (a) the control, (b) the ensemble mean (based on eight cyclic mode members) and (c) control minus ensemble mean. 
barrier $[3,5,6]$; this is also consistent with the coupled ocean-atmosphere instabilities peaking in boreal spring, as shown in Figure 1. Figure 4(c) shows that the ensemble mean forecasts generally perform better than the control forecasts, including during boreal spring.

\section{Conclusions}

We applied ensemble prediction methods, in which the control initial conditions are perturbed by coupled ocean-atmosphere disturbances, within the coupled ocean-atmosphere model of Frederiksen et al. [4]. The bred vectors and cyclic modes have global growth rates of 0.02 day $^{-1}$ corresponding to an e-folding time of 50 days. However, the local growth rates of these perturbations are not uniform but vary with time. We argued that this is the cause of the seasonal variablity in forecast skill.

We performed one year control and ensemble forecasts with the coupled ocean-atmosphere model. The control forecasts were initiated on the first day of each month between 1 January 1980 and 1 December 2000 from analyses obtained using the nudging method of data assimilation. For the ensemble forecasts we considered 2, 8, 32 and 64 perturbations. We focused on the skill of predicting the $50 \mathrm{~m}$ ocean temperature for the NINO3+ region. We find that for forecasts longer than about two months the root mean square errors in the ensemble mean forecasts are smaller than for the control based on averages over all the forecasts started between 1 January 1980 and 1 December 2000. There is considerable variability in the skill of both control and ensemble forecasts during the period studied. This is particularly the case during the period between the start of the El Niño event of 1997 and the La Niña of 1998. Ensemble mean forecast skill improves with increasing number of perturbations but the improvement saturates for larger number of members, with little additional gain in using 64 members compared with 32. We found that ensemble mean forecasts based on eight cyclic modes are more skilful in two to eight month forecasts than those based on 32 to 64 bred vectors. 


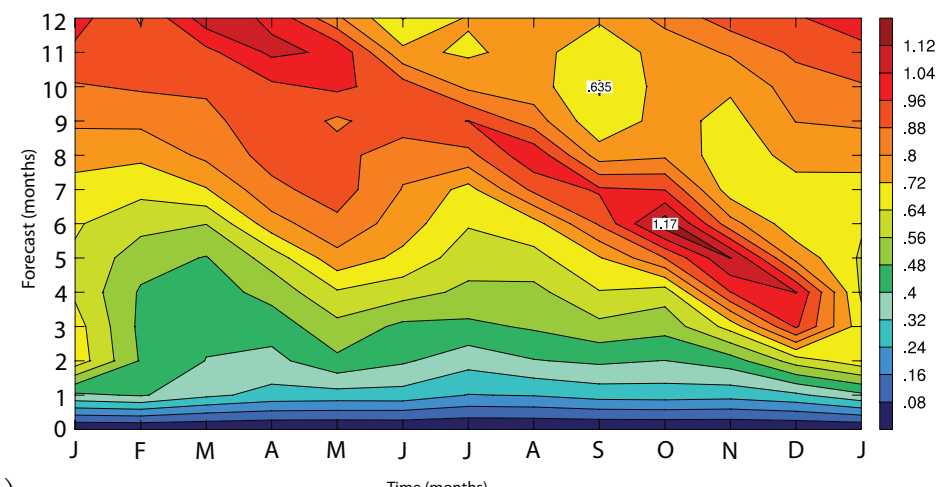

(a)

Time (months)

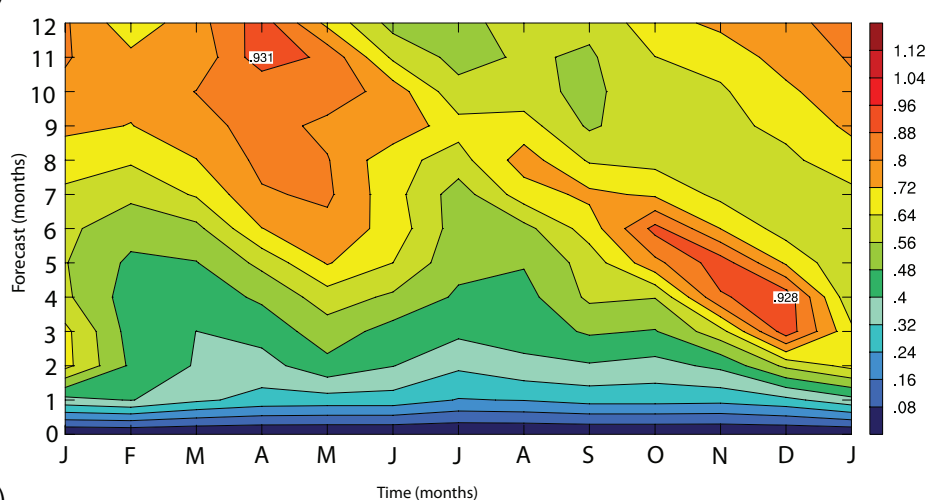

(b)

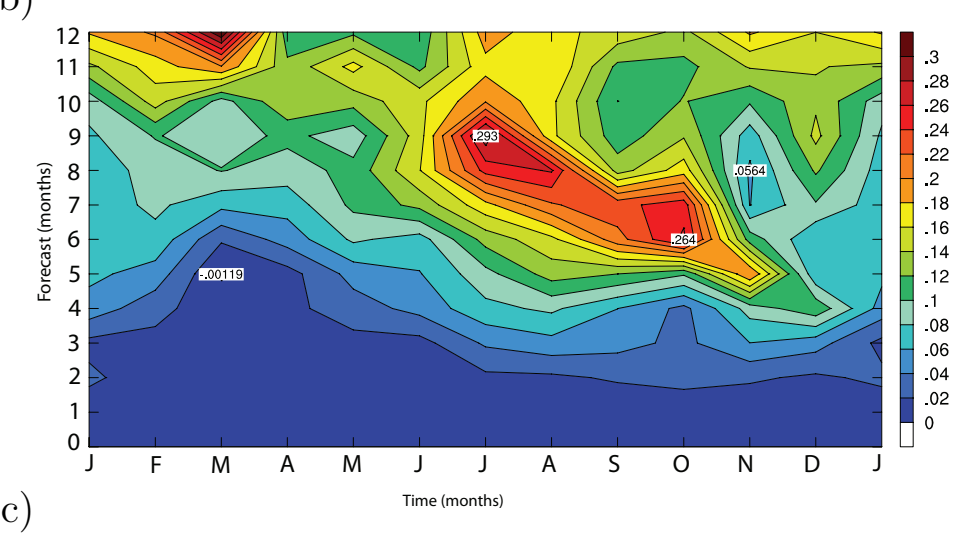

Figure 4: As in Figure 3 but for averages of all forecast errors in a given month. 
We argued, on the basis of the results of Frederiksen and Branstator [5, 6] and our findings in this article, that we expect a contributing cause of the boreal spring predictability barrier is that large scale atmospheric teleconnection patterns and instabilities peak in boreal spring.

\section{References}

[1] Z. Toth and E. Kalnay, Ensemble forecasts at NCEP and the breeding method, Mon. Wea. Rev. 125:3297-3319, 1997. doi:10.1175/1520-0493(1997)125<3297:EFANAT>2.0.CO;2 C363, C364

[2] J. S. Frederiksen, M. A. Collier and A. B. Watkins, Ensemble prediction of blocking regime transitions, Tellus A 56:485-500, 2004. doi:10.1111/j.1600-0870.2004.00075.x C363, C364

[3] S. C. Yang, M. Cai, E. Kalnay, M. Reinecker, G. Yuan and Z. Toth, ENSO bred vectors in coupled ocean-atmosphere general circulation models, J. Clim.,19:1422-1436, 2006, . doi:10.1175/JCLI3696.1 C363, C364, C373

[4] C. S. Frederiksen, J. S. Frederiksen and R. C. Balgovind. Dynamical variability and seasonal predictability in an intermediate complexity coupled ocean-atmosphere model. ANZIAM J. 54:C34-C55, 2013. http://journal austms.org.au/ojs/index.php/ANZIAMJ/article/ view/6296 C363, C366, C367, C373

[5] J. S. Frederiksen and G. Branstator, Seasonal and intraseasonal variability of large-scale barotropic modes, J. Atmos. Sci. 58:50-69, 2000. doi:10.1175/1520-0469(2001)058<0050:SAIVOL > 2.0.CO;2 C363, C367, C373, C375

[6] J. S. Frederiksen and G. Branstator, Seasonal variability of teleconnection patterns, J. Atmos. Sci. 62:1346-1365, 2005. doi:10.1175/JAS3405.1 C363, C367, C373, C375 


\section{Author addresses}

1. J. S. Frederiksen, Climate Adaptation Flagship, CSIRO Marine and Atmospheric Research, Aspendale, Victoria 3195, Australia. mailto: jorgen.frederiksen@csiro.au

2. C. S. Frederiksen, Centre for Australian Weather and Climate Research (CAWCR), Bureau of Meteorology, Melbourne, Victoria 3001, Australia.

3. S. L. Osbrough, CAWCR, CSIRO Marine and Atmospheric Research, Aspendale, Victoria 3195, Australia. 